Adv. Studies Theor. Phys., Vol. 7, 2013, no. 16, 773 - 777

HIKARI Ltd, www.m-hikari.com

http://dx.doi.org/10.12988/astp.2013.3777

\title{
Noise-Induced Excitability of the Complex Liquid Flows
}

\author{
Irina Bashkirtseva
}

Ural Federal University, Ekaterinburg, Russia

Copyright (c) 2013 Irina Bashkirtseva. This is an open access article distributed under the Creative Commons Attribution License, which permits unrestricted use, distribution, and reproduction in any medium, provided the original work is properly cited.

\begin{abstract}
We study an excitability for the stochastically forced system modeling a dynamics of the complex liquid flows. A phenomenon of noiseinduced generation of large-amplitude oscillations in a zone of stable equilibria is studied.
\end{abstract}

Keywords: Excitability, stochastic disturbances, complex liquid flows

\section{Deterministic model}

Consider a complex liquid flow bounded by two parallel planes $z=0, z=$ $h$. The lower plane $z=0$ is fixed and the unidirectional shear stress $\Sigma$ is applied to the upper plane $z=h$. Due to the symmetry, one can limit by one space variable $z$. The physical state of the system is uniquely determined by the function of the viscous stress $\sigma(t, z)$. This function in $[0, \infty) \times[0, h]$ satisfies the equation [1]

$$
\rho \frac{\partial}{\partial t}\left(f(\sigma)+G \frac{\partial \sigma}{\partial t}\right)=\frac{\partial^{2} \sigma}{\partial z^{2}}
$$

with boundary conditions

$$
\sigma(t, h)=\Sigma, \quad \frac{\partial \sigma}{\partial z}(t, 0)=0 .
$$

Here, the function $f(\sigma)$ reflects the nonlinear N-shaped character of the shear rate as a function of the stress (see Fig.1), $\rho$ is a medium density, $G$ is a relaxation parameter. 


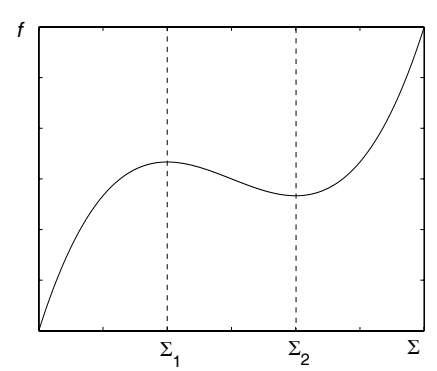

Fig.1. Plot of the function $f(\Sigma)$.

In this paper, for the distributed model of the flow stream (1)-(2) a threelayer discretization is used. For lines $z=z_{i}\left(z_{0}=0, z_{1}=\frac{h}{2}, z_{2}=h\right)$ consider corresponding approximations $\sigma_{i}(t)$ for functions $\sigma\left(t, z_{i}\right)$. Using formulas of numerical differentiation, one get the following approximation of the equation (1) on the line $z=z_{1}$

$$
\rho\left(\frac{d f\left(\sigma_{1}\right)}{d \sigma} \frac{d \sigma_{1}}{d t}+G \frac{d^{2} \sigma_{1}}{d t^{2}}\right)=4 \frac{\sigma_{0}(t)-2 \sigma_{1}(t)+\sigma_{2}(t)}{h^{2}} .
$$

It follows from boundary conditions (2) that

$$
\sigma_{2}(t)=\Sigma, \sigma_{1}(t)-\sigma_{0}(t)=0 .
$$

Equations (3),(4) imply

$$
G \rho \frac{d^{2} \sigma_{1}}{d t}+\rho f^{\prime}\left(\sigma_{1}\right) \frac{d \sigma_{1}}{d t}=4 \frac{\Sigma-\sigma_{1}}{h^{2}} .
$$

A solution $\sigma_{1}(t) \equiv \Sigma$ of the equation (5) is a unique equilibrium. The equation (5) for the variables $x=\sigma_{1}, y=\frac{d \sigma_{1}}{d t}$ can be rewritten as a system

$$
\dot{x}=y, \quad \dot{y}=-\frac{4}{G \rho h^{2}} x-\frac{f^{\prime}(x)}{G} y+\frac{4}{G \rho h^{2}} \Sigma .
$$

For a study of the possible dynamical regimes of this system we fix parameters $G=\rho=h=1, f(x)=k\left(x^{3} / 3-x^{2}+x / 2\right)$. Here, $f^{\prime}(x)=k\left(x^{2}-3 x+2\right)$. The function $f(x)$ models a characteristic type of N-shaped curve mentioned above, and the parameter $k$ reflects a stiffness of this nonlinearity.

The equilibrium $x=\Sigma, y=0$ of the system (6) is stable for $0<\Sigma<1$ and $\Sigma>2$. On the interval $1<\Sigma<2$, this equilibrium is unstable and a stable limit cycle is observed. The extreme values of the variable $x$ for attractors of the system (equilibria and cycles) on the interval $0<\Sigma<3$ for $k=1,20$ are plotted. The instability of the equilibrium for $\Sigma \in(1,2)$ leads to the appearance of the large-amplitude auto-oscillations of the flow. 
a)

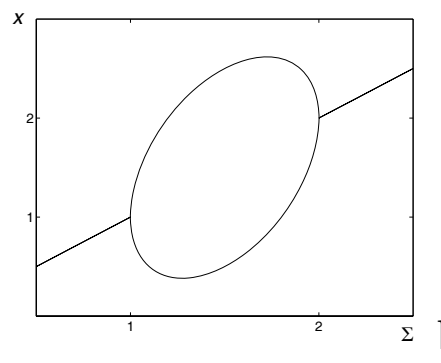

b)

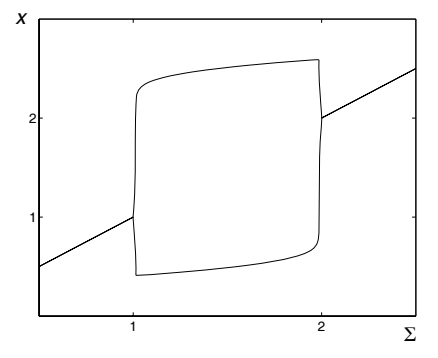

Fig.2. Extreme values $x$ for the attractors: a) $k=1$, b) $k=20$.

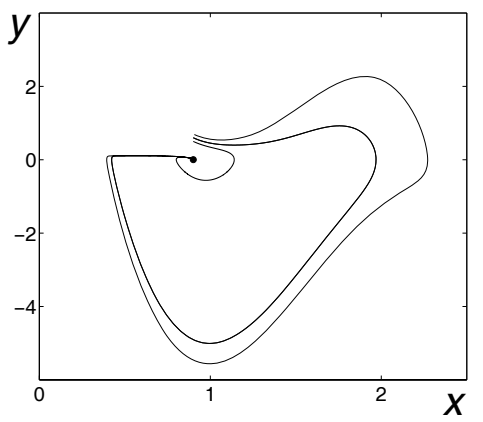

Fig.3. Phase portrait of the deterministic system (6) for $\Sigma=0.9$ and $k=20$.

In this paper, we focus on the zone $\Sigma<1$ close to the point $\Sigma_{1}=1$ of Andronov-Hopf bifurcation. In Fig.3, a phase portrait of the deterministic system (6) for $\Sigma=0.9$ is plotted. Small deviations of the initial state from the equilibrium result in small-amplitude trajectories that correspond to subthreshold responses. If we take initial deviations larger than some threshold, large-amplitude trajectories appear that correspond to suprathreshold response. Around the equilibrium, one can find a set of initial points corresponding to the subthreshold response. A size of this subthreshold domain essentially depends on the parameter $\Sigma$. The closer $\Sigma$ to the bifurcation value, the less a size of this subthreshold domain.

Such non-uniformity of the phase portrait is a underlying reason of the stochastic excitability of the studied system. Noise-induced excitability was studied for various dynamical systems [2-5].

\section{Stochastic model}

For the analysis of stochastic effects, consider the randomly forced system

$$
\dot{x}=y, \quad \dot{y}=-4 x-k\left(x^{2}-3 x+2\right) y+4 \Sigma+\varepsilon \dot{w}(t),
$$

where $w(t)$ is a standard Wiener process, $\varepsilon$ is a noise intensity.

In Figs.4-5, random trajectories and time series of the system (7) for two values of the noise intensity are plotted. For weak noise $\varepsilon=0.1$, random trajectories leave the stable equilibrium and concentrate in the subthreshold zone. 
a)

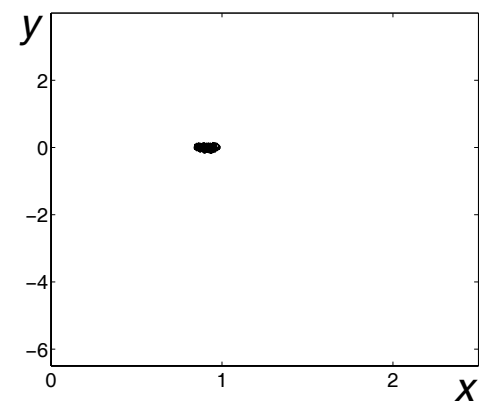

Fig.4. Random trajectories of the system

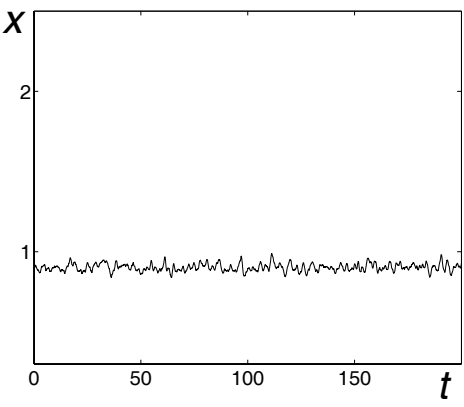

Fig.5. Random time series: b)

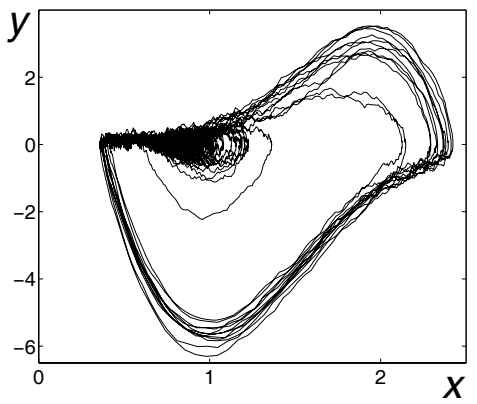

b)

: a) for $\varepsilon=0.1$, b) for $\varepsilon=0.5$.

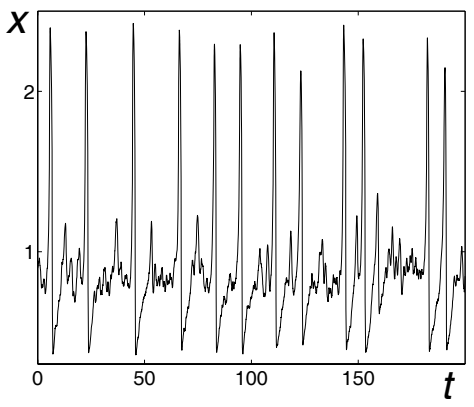

a) for $\varepsilon=0.1$, b) for $\varepsilon=0.5$.

Here, time series demonstrate small-amplitude stochastic oscillations near the equilibrium (see Fig.5a). As the noise intensity increases, random trajectories transit to the suprathreshold zone. As one can see in Fig. $4 \mathrm{~b}$ for $\varepsilon=0.5$, the system exhibits stochastic oscillations of large amplitude. An intermittency of small- and large-amplitude oscillations is clearly seen in Fig.5b. So, this system is highly excitable to stochastic disturbances. This model exhibits a noise-induced stochastic cycle even when the deterministic system has a stable equilibrium only.

Acknowledgements. The study was supported by the Ministry of education and science of Russia, project 14.A18.21.0364.

\section{References}

1. I.A. Bashkirtseva, A.Y.Zubarev, L.Y. Iskakova, L.B. Ryashko, On rheophysics of high-concentrated suspensions. Colloid Journal, 71 (2009), 446-454.

2. S. Fedotov, I. Bashkirtseva, L. Ryashko, Stochastic analysis of a nonnormal dynamical system mimicking a laminar-to-turbulent subcritical transition. Phys. Rev. E, 66 (2002), 066310.

3. B. Lindner, J. Garcia-Ojalvo, A. Neiman, L. Schimansky-Geier, Effects of noise in excitable systems. Phys. Rep., 392 (2004), 321-424. 
4. L. Ryashko, I. Bashkirtseva, Analysis of excitability for the FitzHughNagumo model via a stochastic sensitivity function technique. Phys. Rev. E, 83 (2011), 061109.

5. I. Bashkirtseva, A. B. Neiman, L. Ryashko, Stochastic sensitivity analysis of the noise-induced excitability in a model of a hair bundle. Phys. Rev. E, 87 (2013), 052711.

Received: July 11, 2013 